\title{
Analysis of the Evaluating Modes of the Liberal Arts Collaborative Innovation Centre
}

\author{
Jie Yang, Qiliang Pan* \\ Social Science Research Department of Jinan University, Guangzhou \\ Guangdong, 510632, China
}

\begin{abstract}
Based on the characteristics of the liberal arts collaborative innovation centre, this paper reveals a series of problems existing in the evaluation methods of the liberal arts collaborative innovation centre from four aspects: evaluation organization, evaluation cycle and process, evaluation index system and evaluation results. The author puts forward his own suggestions on how to construct an evaluation method that meets the characteristics of the liberal arts collaborative innovation centre.
\end{abstract}

Keywords: collaborative innovation centre, evaluation method, construction

\section{Research Background}

According to the new requirements of the party and the state on the development of higher education in the new situation, in 2012 the Ministry of Education and the Ministry of Finance issued the Opinions on Implementing the Plan for Enhancing the Innovative Abilities of Institutions of Higher Learning (abbreviated as "2011 Plan"). This is our country higher education following the "985", "211" project, another embodiment of the national will of the strategic plan, it is to improve the quality of education, personnel training, improve university internal resources and external innovation forces for the purpose of scientific and technological system reform "Universities, human resources, scientific research," the trinity of the capacity to enhance the model is the country's strategy of revitalizing the country through science and education and talent strategy is another important embodiment. 
To implement the "2011 Plan", the Ministry of Education in 2013 launched the "2011 Collaborative Innovation Centre" that work. At present, two batches of 38 national-level collaborative innovation centres have been identified, including 7 liberal arts collaborative innovation centres. The leading universities are China University of Political Science and Law, Nanjing University, Tianjin University, Beijing Normal University, Fudan University, Xiamen University and Tsinghua University. In addition to national-level collaborative innovation centres, many provinces and even universities themselves have identified a number of provincial or university-level collaborative innovation centres, many of them liberal arts collaborative innovation centre.

\section{The necessity to build scientific evaluation methods of liberal arts collaborative innovation centre}

Collaborative Innovation Centre is an important carrier of the "Plan for Enhancing the Innovative Ability of Colleges and Universities" (the "2011 Plan"), which is divided into four types, which are science-oriented, culture-oriented, industry-oriented and regional-oriented. Of the collaborative innovation centre to philosophy and social science as the main body, the other three types of collaborative innovation centre to science and engineering as the main body. "2011 Plan", the Liberal Arts Collaborative Innovation Centre and the Science and Technology Innovation Centre is the positioning of collaborative innovation, liberal arts mainly to enhance the national cultural soft power and enhance the international influence of Chinese culture as the goal, focusing on social benefits; Is to promote the progress and development of science and technology, knowledge innovation, technological innovation, focusing on scientific and economic benefits [2].

\section{The evaluation status quo and existing problems of the collaborative innovation centre of liberal arts}

Because the country implemented the "2011 plan" for a short period of time, although there are some corresponding measures and methods to formulate and promulgate some relevant documents for the evaluation method of the $\mathrm{CCC}$, it can be seen that neither the "accreditation method" "Declaration", and "expert feedback" are still broad-brush, impression, and even the views of individual experts or emotional, has a strong emotional, it is difficult to different types of collaborative innovation centre to make more accurate and objective And the right evaluation and judgment. Especially for the liberal arts collaborative innovation centre, so far the constructions, selection and acceptance, as well as the development of evaluation indicators, is still in a trial and trial stage, there are still many problems. 


\subsection{Evaluation organizations}

The design of the evaluation organization will not only lead to the evaluation of fairness and justice, but also will lead to the evaluation of whether the scientific, professional, different research fields, different disciplines, different research types (based, application-based, fully applied), Its evaluation of organizational structure should be differentiated. But for the time being, the evaluation organization of liberal arts collaborative innovation centres has long been dominated by government departments, and lack of diversified evaluation bodies. In the evaluation of liberal arts collaborative innovation centres, evaluation content, evaluation time, evaluation methods and evaluation results And so on depend on the government departments and administrative intervention is too heavy, not conducive to both liberal arts collaborative innovation centre diversity and complexity. At the same time, China's colleges and universities are the main force of the humanities and social sciences research, although the relevant documents explicitly mentioned by the third party to organize evaluation, but the relative lack of intermediaries, in practice, the evaluation of the members of the organization more by the experts. Assessment experts from the "single", will liberal arts collaborative innovation centre closed, departure from the original intention.

\subsection{The evaluation cycle and process}

"2011 Collaborative Innovation Centre" both a construction process, but also a declaration that the work, there is a task to complete the experience of different processes have different priorities, how to evaluate and assess the efficiency of various processes, to establish what Like the evaluation cycle, which are urgent problems to be solved. According to the MOE document, the CCC uses a threeyear evaluation cycle, but it does not specify what the annual assessment is and how comprehensive it is each year. If the former, will lead to over-evaluation, so that the collaborative innovation centres struggling to cope with checks, increasing the burden?

\subsection{The evaluation index system}

The evaluation system and the index system of the liberal arts collaborative innovation centre are the core. Scientific and reasonable evaluation index system, is an important guarantee for the success of the construction of collaborative innovation centre of liberal arts. The existing collaborative innovation centre evaluation index system mostly emphasizes the characteristics of natural science research, while ignoring the disciplines of humanities and natural science differences, and the disciplines of the humanities disciplines, disciplinary differences, it is difficult to use a unified evaluation System "to beat the world"; the same time, the current widely used evaluation index system more emphasis on quantitative indicators, too much emphasis on project level, the size of funding and publication of the level of publications, while ignoring the humanities and social science research academic level and social contribution; At 
the same time, some of the evaluation indicators too emphasize the short-term benefits of humanities and social sciences, ignoring the humanities and social sciences academic research long-term accumulation process and long-term development evaluation. The evaluation index system is not perfect, the evaluation index is simple, the evaluation criteria are quantified, and so on, these problems will hinder the cooperative innovation. How to establish the evaluation index system in line with the characteristics of humanities and social science disciplines and scientific research in the selection, construction and acceptance of collaborative innovation centre is of great significance to promote the construction and development of collaborative innovation of liberal arts.

\subsection{The application of evaluation results}

"Evaluation is the process of providing useful information for decision-making," says Stafelbim, who believes that "the most important intention of the evaluation is not to prove, but to improve." For now, the Collaborative Innovation Centre Most of them are single, and the peer assessment experts can only evaluate the overall situation of each CCC according to the evaluation requirements and standards, and classify the evaluation results as excellent, good, general and poor, and evaluate the results. Although there are some comments on the specific evaluation indicators, but on the whole, the centre has made progress in the construction, there are still some specific problems such as the lack of more targeted description, "between the evaluation results and the evaluation of the object is not Even if it is understood by the research institution, whether the research institution has made the evaluation according to the opinions of the evaluation institution, and whether the evaluation result is in accordance with the opinion of the evaluation institution, whether it can be understood by the research institution or not. The corresponding adjustment is still unknown "[3], leading to the evaluation of the collaborative centre of the next step in the direction of efforts to understand is not clear, is not conducive to the implementation of rectification opinions.

\section{The construction of scientific evaluation methods of liberal arts collaborative innovation centre}

\subsection{Construct management-evaluation separation, relatively independent evaluation organization}

Evaluating organization is the main body and key of evaluation method. The design of evaluation organization will not only result in fairness and justice, but also will result in evaluation whether scientific and professional. Different research areas, different disciplines, different types of research, its evaluation of organizational composition should be differentiated. The "2011 Plan" will be organized into management and operational level, management, namely the Ministry of Education, Ministry of Finance jointly established the "2011 Plan" 
leading group, as well as from the relevant departments, universities, research institutions, industry, Experts from social groups form an expert advisory committee. The management layer is responsible for coordinating innovation centre identification and the establishment of performance evaluation system and the publicity and application of evaluation results. The operation layer is the third-party evaluation organization, which is responsible for selecting and appraising experts, conducting evaluation and conducting periodic inspections. The construction of evaluation organization mode of liberal arts collaborative innovation centre should reflect the independence, professionalism, fairness and openness, and construct the evaluation mechanism of separation of management and evaluation. Specifically, it includes the following three aspects:

The Evaluation Leading Group consists of the Integrated Evaluation Team, the Program Implementation Evaluation Team, and the Evaluation and Evaluation Team. The Evaluation Team is responsible for the evaluation of the CICC's integrated services, institutional mechanisms and implementation. The Evaluation Team is responsible for co- Evaluation of the Centre's operations and internal management; and the use of the Evaluation Team to evaluate the collaborative innovation centres of different disciplines and types according to the different areas and into different groups.

The selection of evaluation experts will take the form of gradually reducing the number of personnel from universities, increasing the number of personnel from industry, government and civil research institutions and maintaining appropriate numbers. Due to the differences in the knowledge structure, working background and way of thinking of different sources, the viewpoints of the centre's achievements and operational mechanism will differ to some extent. They will explore and coordinate with each other and make suggestions to the National Demand, Collaborative Innovation Centre The direction of the development, the setting of the evaluation focus has a scientific guiding role, which is conducive to ensuring the objectivity of the evaluation.

And the evaluation of the main non-interest relationship between the independent third parties to ensure that the liberal arts collaborative innovation centre evaluation of the efficiency of justice has an important role in protection. Third party evaluation can effectively run, the key in the construction of expert library, "according to different disciplines and industry categories were set up academic experts, practical experts, and according to different types of collaborative innovation centre features, adjust the proportion of expert group structure, Follow the random, avoid the principle of selection "[4]. At the same time, we should attach great importance to the "academic prestige" and "moral morality" of the storage experts, which will help to resist the academic corruption.

\subsection{Build a comprehensive three-dimensional, classified guidance evaluation index system}

Indicators are the basic elements of the evaluation, the index system refers to the principle according to certain indicators to filter and assign its weight. The establishment of a scientific and reasonable evaluation index system is the core of 
the implementation of evaluation, and for the evaluation index of collaborative innovation centre, "should focus on 'innovation' and 'synergistic force' two dimensions. The synergetic dimension of the collaborative innovation centre should focus on the management operation and open sharing of the collaborative innovation centre. "[5] The synergetic dimension of the collaborative innovation centre is reflected in the scientific frontier, cultural heritage, industry and regional development.

There are different structures and contents of the appraisal evaluation, the midterm construction evaluation and the final evaluation, if the evaluation object is different, that is, the evaluation, the mid-term construction evaluation and the node evaluation. The most effective way is to establish the appraisal index system firstly, and then adjust the structure and content of the evaluation index system according to the different requirements to form the evaluation index system of mid-term construction evaluation and outcome. Based on the research of the evaluation index system of liberal arts collaborative innovation centre and the actual situation of the declaration and identification work of the National and Guangdong Collaborative Innovation Centre in recent one or two years, this paper first tries to construct the evaluation index system of the collaborative innovation centre of liberal arts. The index is constructed according to the principle of classification guidance, which is divided into three grades. One index is relatively abstract, followed by step by step more and more specific, the most specific indicators of the final level. In our design of the index system, a target is divided into collaborative needs, synergies, coordination mechanisms, synergies, and then on the basis of specific divided into two indicators and three indicators. For example, in the coordination mechanism of this level of indicators, the separation of organizational model, mode of operation, management system, these three secondary indicators, and finally in the secondary indicators on the way, and can be separated from the operational language of the general expression of the three Indicators, such as the establishment of the Council under the leadership of the centre director responsibility system, personnel appointment, task assignment, resource deployment, performance evaluation, pay distribution and other three indicators. The evaluation index can be expressed in a more comprehensive, systematic and complete way, which can be used to evaluate the collaborative innovation centre of liberal arts. After the establishment of the evaluation index, we also made a questionnaire on the selection of the evaluation index system of the collaborative innovation centre for liberal arts, and sent out 634 questionnaires to the universities and research institutes, and actually recovered 612 copies.

$96 \%$ of the questionnaires agree with the establishment of a secondary index, in the establishment of the three indicators, $76 \%$ of people fully recognized the establishment of the index, $18 \%$ of the indicators that can be part of the need to modify, Such as the first 19 indicators, a questionnaire that can, but proposed to address the urgent need to address economic and social development to solve major problems and livelihood issues, the indicators were added. Another small part of the questionnaire, the three indicators are not recognized attitude, such as the first 57 indicators and proposed changes to the performance appraisal method. 
From the questionnaire results, most of the scientific research managers of the establishment of the three indicators are recognized attitude. The construction of the mid-term construction evaluation and outcome evaluation index system of liberal arts collaborative innovation centre can be adjusted on the basis of the evaluation index system and the specific practical work of evaluation.

\subsection{Construct a dynamic evaluation method combining self-assessment, sampling and comprehensive assessment}

Collaborative Innovation Centre is a multi-dimensional, open and dynamic organization, and its collaborative innovation activities are a long-term complex systems engineering, involving the re-configuration of capital, personnel, information and other innovative elements and a series of re-design of institutional mechanisms. Evaluation of its performance and evaluation of previous scientific research is different, in addition to examining the extent to achieve the desired goal, that is, the evaluation of its final results, but also pay attention to the process of intervention and regulation to prevent the listing of its poor running or not running phenomenon. At present, the liberal arts collaborative innovation centre is basically a four-year construction cycle, fouryear period will take the exit mechanism of survival of the fittest. Therefore, it should be stipulated that the Centre for Liberal Arts Collaboration and Innovation shall formulate a 4-year development strategy plan and formulate annual work plans that can be quantified year by year, and carry out annual self-evaluation on this basis and report to the self-assessment report. "Self-evaluation should include two levels of content, first, collaborative innovation centre in the absence of external factors involved in the case, step by step to carry out internal selfevaluation; Second, collaborative innovation and internal institutions and related personnel self-evaluation". Through the way of self-assessment, not only can the liberal arts collaborative innovation staff understand their progress, achievements and problems in the development, such as the management system, personnel system and other institutional mechanisms, but also allow the relevant evaluation agencies more detailed To understand the specific details of the operation of the collaborative innovation centre, and reveals common problems for the follow-up of the liberal arts collaborative innovation work to put forward more guidance.

\subsection{Construct a more concrete and pragmatic result evaluation method}

The core of the results evaluation mode lies in the form of publication of results. This paper argues that, in order to achieve the purpose of evaluation, the content of the current evaluation results should be more informative and diversified, in addition to listing the level of collaborative innovation centre. We should pay attention to the follow-up evaluation and process evaluation to the CCC, and add the following contents in the evaluation results to make the CICS innovation results more scientific and reasonable. "It is necessary to include appropriate textual descriptions and explanations of strengths and weaknesses so that the Centre for Collaborative Innovation can be fully understood. Secondly, to build a coherent, consistent results evaluation system. The results of any evaluation 
cannot be single, isolated, and therefore to establish a coherent, dynamic results evaluation system, the results of multiple evaluations to take into account and absorb the adoption, and focus on the centre of the problems and the corresponding corrective measures, the formation of evaluation results and evaluation of the effective interface between the object, in order to maximize the role of the results of evaluation. Collaborative innovation centre should not only colleges and universities, research institutions, "one-man show", to encourage competition and the evaluation results of the centre should be timely to the same industry, other scientific research institutions.

\section{Acknowledgements}

This paper is the stage result of the Education Department of Guangdong Province project "liberal arts collaborative innovation center evaluation system" (2013zczx0003).

\section{References}

[1] Zhao Jianmei, Liu Yun .Research on the Publication and Application of the Evaluation Results of Scientific Research Institutions. Science \& Technology Forum, 55(12), pp.68-70, 2011

[2] Ma Weihua. Research on Evaluation Problem in the Field of Collaborative Innovation. Higher Education Exploration, 8 (5), pp.87- 91, 2013

[3] Zhao Dewu. Construction of Performance Evaluation System of Collaborative Innovation Center, Chinese University Technology, 12(10), pp. 18- 20, 2012

[4] Zhu Peng, Pan Lin. Study on the Evaluation System of Collaborative Innovation Center. Journal of Henan Normal University, 9(6), pp.58- 61, 2011

[5] Zhao Jianmei. Liu Yun. Discussion and Application of Evaluation Results of Scientific Research Institutions. Technology Forum, 8(4), pp.85-87, 2014 